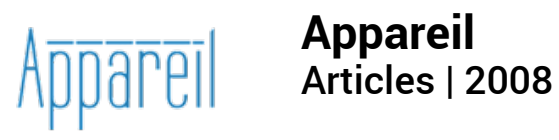

\title{
À bon entendeur
}

Jean Lauxerois

\section{(2) OpenEdition \\ Journals}

\section{Electronic version}

URL: http://journals.openedition.org/appareil/111

DOI: 10.4000/appareil. 111

ISSN: 2101-0714

\section{Publisher}

MSH Paris Nord

\section{Electronic reference}

Jean Lauxerois, « À bon entendeur », Appareil [Online], Articles, Online since 09 February 2008,

connection on 30 July 2020. URL : http://journals.openedition.org/appareil/111 ; DOI : https://doi.org/ 10.4000/appareil. 111

This text was automatically generated on 30 July 2020 .

\section{(c) (i) (9)}

Appareil est mis à disposition selon les termes de la Licence Creative Commons Attribution - Pas d'Utilisation Commerciale - Pas de Modification 4.0 International. 


\title{
À bon entendeur
}

\author{
Jean Lauxerois
}

(texte initialement paru dans la revue Circuit, Volume 14, numéro 1 (2003), Presses de l'Université de Montréal)

1 Il est curieux de voir combien le concept adornien dit d'“ écoute structurelle ", tel qu'il apparaît dans Introduction à la sociologie de la musique, peut servir de repoussoir obligé à tant de considérations bien entendues sur l'écoute musicale. Il n'est pas rare de voir poindre, ici et là, le ouï-dire de la vulgate, qui propose bien souvent, contre cette théorie dite "monolithique" et "purement réactive", une autre écoute jugée évidemment "moins directionnelle". Certes, il est bien légitime de récuser toute perspective normative, privilégiant l'orthodoxie d'une écoute qui se voudrait analyse et synthèse à la fois, pour s'imaginer devenir adéquate à ce que l'œuvre lui aurait déjà prescrit; impossible de réduire le phénomène de l'écoute musicale à cette écoute prétendument experte au nom de laquelle toutes les autres formes d'écoute se verraient renvoyées à leur insuffisance ou à leur nullité; impossible de ne pas lui opposer la plasticité féconde de ces autres écoutes qui, de la distraction à tous les modes d'arrangement, laisseraient libre champ à la finitude des signatures subjectives. Mais s'en tenir à la seule Introduction à la sociologie de la musique pour juger de la pensée d'Adorno, c'est raisonner un peu court, parce que c'est faire l'impasse sur des textes importants des années soixante, la plupart encore non traduits, où cette pensée se métamorphose et dont l'éventail enrichit, voire modifie singulièrement la perspective.

On découvre, en effet, non seulement qu'Adorno ne fait pas de l'écoute structurelle son credo définitif, mais surtout qu'il construit moins une théorie de l'écoute qu'une pensée de l'ouï, impliquant une pensée neuve de l'œuvre d'art. Loin de se référer à une métaphysique du "sujet" et à une idée esthétique de l'œuvre, implicites fondements de toute théorie de l'écoute, Adorno pense l'œuvre d'art sous le signe de la technè, entendue comme technique de reproduction, comme technique de construction et comme technique de production du temps : ce qui engage du même coup à reconsidérer qu'il y aurait une idéalité, une essence invariante de l'écoute, qui serait celle d'un "sujet" lui-même invariant. Si l'on conviendra, dès lors, qu'il y a bien 
une historicité de nos oreilles, ce serait pour poser que cette historicité est avant tout celle du concept même d'écoute. À cet égard, la promotion moderne du terme, dont le sens actuel remonte juste au début $\mathrm{du} \mathrm{xx}^{\mathrm{e}}$ siècle, correspondrait au destin métaphysique et historique de l'homme comme "sujet", aux prises avec ce qu'il faudrait nommer son oto-nomie, symptôme d'un "mal d'oreille" ou d'un "malaise dans l'écoute ", qui serait l'un des modes de celui que Freud explore dans la culture.

L'opiniâtre surdité à la pensée d'Adorno a ses raisons particulières, mais elle n'est peutêtre pas étrangère à ce " malaise ". On peut s'étonner notamment qu'au moment où l'on refuse, à juste titre, les implications évoquées de l'écoute structurelle, on souscrive implicitement, et quoiqu'on s'en défende, à une idée de l'œuvre qui apparait elle-même souvent prescrite autant que préjugée, et on accorde à l'écouteur un privilège qui sousentend, au nom de cette finitude, une subjectivité étrangement omnipotente autant que naïvement indiscutée. Car l'enjeu est bien celui d'un "sujet" autonome au point d'en être sourd à son radical défaut : 1 '“ otonomie " de l'écouteur le condamnerait à ne pas entendre la finitude de son écoute, parce qu'il serait pour ainsi dire branché sur l'Écho de Narcisse. Cette finitude est pourtant au cœur de ce qui porte la différence des verbes de notre oreille; c'est elle qui fracture l'audition, partagée entre l'ouïr, l'entendre et l'écouter ; c'est elle encore qui met notre oreille en prise sur le langage, sur la voix, sur le son et sur le silence, alors que l"“ oto-nomie " du sujet l'inclinerait à l'écoute prétendument singulière voire isolée du son. Entre auscultare, intendere et audire, entre l'écoute, l'audition, l'entente et l'ouie, entre l'écouteur, l'auditeur et l'entendeur, de même d'ailleurs qu'entre l'acoustique, le sonore, et le musical, il y a différence, et différence de(s) sens. À se demander même s'il y a une écoute "proprement" et expressément musicale, puisque l'ouïr relève d'abord, au défaut du "sujet" et de sa finitude, d'une temporalité dont La Recherche du temps perdu montre qu'elle est à la fois le cœur et la clef du fait que nous entendons, voyons et comprenons toujours à l'envers. Et comme Proust l'expérimente avec la musique de Vinteuil, l'“ ouïr" qui s'élabore temporellement implique la mutabilité infinie d'un sujet qui se résorbe et se construit dans l'œuvre. Pour esquisser manière de réponse à la question “Qui écoute?", tentons d'entrer dans ce multiple défaut d'oreille. Sans baisser pavillon.

\section{Mots d'oreille, en vestibule}

Laissant de côté l'argument d'Introduction à la sociologie de la musique, commençons donc par entendre, c'est-à-dire par prêter l'oreille à la langue, car la formule même de dasstrukturelle Hören ne laisse pas d'intriguer dès lors qu'elle devient dans sa traduction française "l'écoute structurelle ". La différence entre le français et l'allemand a de quoi nous alerter, parce qu'il nous faire entendre, dans la différence des langues, une autre différence : celle qui habite et constitue le registre de notre oreille.

La question se pose de manière cruciale, parce qu' écouter se dit en allemand zuhören, ou anhören, tandis que le verbe simple hören renvoie à l'indistinction de l'entendre et de l'écouter, c'est-à-dire simplement à l'ouï. Or, Adorno emploie toujours le verbe hören, et jamais zuhören ou anhören, pour dire l'“ entendre/écouter" de la musique, quel qu'il soit. Il n'est donc sans doute pas exact de traduire das strukturelle Hören par "l'écoute

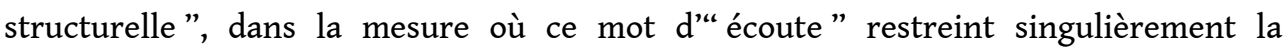
richesse des sens entendus dans le verbe hören. Voilà qui nous reconduit à examiner le 
lexique de la langue française, pour constater que le mot d'“ écoute", qui paraît aujourd'hui aller de soi, est en réalité un produit récent, attestant et relevant d'une profonde mutation - historique, technique, "culturelle".

On sait qu' écouter, qui vient de es/ex/as-coltare < auscultare, a le sens de "écouter avec attention", "ajouter foi", "écouter en cachette"; son doublet est évidemment "ausculter" ( $\left.\mathrm{xvI} \mathrm{I}^{\mathrm{e}}\right)$, au sens initial d'" écouter à des fins médicales". Le substantif l' écoute, au début XII ${ }^{\mathrm{e}}$, signifiait d'abord "celui qui écoute", puis désigna, au Xve siècle, un lieu fermé d'où l'on peut, dans un couvent, suivre l'office sans voir ni être vu. Une écoute désigne aussi, à la même époque, l'action de faire le guet, sens qu'on retrouve dans l'expression "être aux écoutes". Au XIX ${ }^{e}$ siècle (1 864), le terme prend un sens expressément militaire, comme dans le " poste d'écoute ", évoquant la détection, par le son, de l'activité ennemie. Et c'est seulement au début du $x^{e}$ siècle que "l'écoute" prend son sens actuel, en liaison avec le développement des techniques de radiophonie et de téléphonie. Ce sens technique est contemporain de ce que la psychanalyse inaugure à son tour en matière d'écoute, peu à peu banalisée par la psychologie jusque dans la valorisation de l'attitude qui consiste à "être à l'écoute de quelqu'un ", voire, plus récemment, à être - absolument - "à l'écoute ". L'affaire est donc assez neuve, si neuve même que dans la Recherche du temps perdu, par exemple,et même dans les pages nombreuses consacrées à la musique, Proust n'emploie jamais le mot “écoute ", et bien peu le verbe "écouter", sinon dans des moments particuliers, pour dire le rapport de Swann ou de Marcel à l'œuvre de Vinteuil. L'écoute, dans son lexique même et dans son sens contemporain, est donc un fait de culture, supposant la grande mutation technique de toutes les " phonies" de l'“ écouteur", qui va lui permettre de supplanter l'audition, et de reléguer presque l'auditeur au rang des archaïsmes.

7 La différence est évidemment grande qui sépare cette sphère de l'“ écouter " de celle de l'" entendre". Le verbe "entendre", venant de intendere, suppose à l'origine une " intention ", qui faisait de lui l'équivalent de "comprendre " (comme lorsqu'on disait, par exemple, “j'entends l'allemand"); “entendre" avait aussi le sens de "faire attention" et, quasi secondairement, celui d" être frappé d'un son"; or, ce sens devenant dominant a fini par prendre la place du mot "ouïr", qui, lui, vient directement de audire: audir est l'action d'entendre (1080), puis se retrouve sous la forme oïr (1190) dans les expressions oïr dire, oïr parler. Ajoutons à ce panorama les substantifs de la racine de audire : "l'audience " (1160) dit d'abord l'attention à celui qui parle, l'attention favorable, puis est synonyme d'auditoire, pour dire ensuite l'entretien et la séance du tribunal; "l'audition" (1390) est la fonction de l'ouïe, l'action d'entendre, puis la séance musicale ; enfin "l'auditoire " aura d'abord été le lieu où l'on "écoute" puis l'ensemble des personnes qui écoutent. La désuétude de l'ouir, et de tout ce qui a racine dans l'audir, aura successivement laissé la primeur à l'“ entendre ", puis à l'“ écouter ", au sens où nous l'employons aujourd'hui.

8 Entre les langues, donc, certes, mais surtout dans la langue en général, passe la différence des sens, c'est-à-dire la différence d'un sens, puisque ce qui traverse "l'ouïr" relève de plusieurs dimensions. Or, comme le montrent l'histoire de la langue et celle de nos oreilles, cette différence se trouve avoir été neutralisée, au privilège de l'écoute et, à sa suite, d'une écoute dite musicale. Nous prendrons donc "l'écoute " avec les pincettes des guillemets, en guise de speculum introduit dans notre défaut d'oreille. 


\section{L'oreille spéculative}

9 Cette spéculation est bien celle d'Adorno, dès lors qu'on lui reconnaît le mérite d'avoir lui-même profondément modifié son approche de la question, au point de s'expliquer à la lettre sur les raisons de la notion d" écoute structurelle" et de la situer dans sa relativité. Le texte le plus décisif sur ce point s'intitule Schöne Stellen (Beaux passages); il date de 1965, c'est-à-dire de cette époque féconde des dernières années où Adorno continue à instruire, sous forme radicalement fragmentaire, des questions souvent ouvertes de longue main. Ce texte transcrit une émission de radio, dans laquelle il s'adresse aux chers auditeurs, leur donnant à "écouter" à la fois un programme d'extraits d'œuvres - de ces fragments qui forment autant de "beaux passages", de Bach à Schönberg - et des remarques afférentes qui constituent autant d'éléments d'une théorie de l'oreille en musique.

L'enjeu initial du texte est bien de rompre avec le simplisme auquel l'écoute dite structurelle s'est trouvée réduite. Conscient des objections qu'on n'a sans doute pas manqué de lui faire à l'époque, Adorno s'explique d'emblée sur les raisons historiques qui ont guidé sa première approche de la question dans Introduction à la sociologie de la musique : la notion d'écoute structurelle, dit-il, avait avant tout une fin critique et visait à dénoncer cette tendance à l'atomisation et à la passivité naturaliste que commençait à imposer l'industrie culturelle, notamment par le détour de la musique de variétés. L' écoute structurelle " devait faire pièce à cette "écoute atomisée " (das atomistiche Hören) qui devenait alors la règle et le style de l'écoute contemporaine. Pourtant, avance alors Adorno, les choses "se sont renversées" : il s'agit maintenant de récuser tout autant, et à l'opposé, une écoute prétendument cultivée qui devient dominante ; elle est le fait de l'idéal musicologique de type néoclassique et historiciste, qui se réclame d'une objectivité à laquelle Adorno reproche d'être du style "machine à coudre". Sa suffisance est d'autant plus insupportable qu'elle se revendique comme une écoute totale, qui procède d'une erreur radicale sur l'idée de totalité, laquelle menace la musique elle-même. Pareille prétention méconnaît que la totalité n'est pas la somme des parties, et qu'elle n'est pensable que comme processus, comme articulation et jeu du détail. Il ne faut donc pas confondre "écoute structurelle" et "écoute totale". La première s'approfondit comme rapport au détail, et puisque le détail, c'est l'œuvre au travail dans les plus fines de ses articulations, puisque le sens de la totalité n'est audible et n'est lisible qu'au niveau du détail, le "regard" doit désormais savoir perdurer, en s'attardant sur l'élément singulier : telle est, dit Adorno, la dimension complémentaire dont il faut " d'urgence " doter l'“ ouïr structurel " pour lui donner sa signification véritable.

11 Le temps joue ici un rôle décisif. L'“ ouïr " ne peut désormais s'effectuer ni selon le flux ni selon l'instant atomisé, mais dans le seul temps du discontinu. Le mot ne se trouve pas à la lettre dans le texte d'Adorno, mais se déduit aisément de la logique de son argumentation. Il est le point par où la musique approche au plus près la manière dont bascule la question du temps au $\mathrm{xx}^{\mathrm{e}}$ siècle : le temps ne peut plus être réduit à cette continuité linéaire dont la fiction de l'instant ponctuel est le malheureux corollaire. Zum richtigen Hören, pour entendre " de manière droite ", il faut entrer dans le mode de la discontinuité. C'est cette discontinuité qui permet aussi de penser la fracture entre détail et totalité, selon un paradoxe qui n'est évidemment qu'apparent : du détail à la structure en effet, il n'y a en vérité pas d'adéquation possible; nous pouvons, nous 
devons l'entendre ainsi, parce que nous sommes les héritiers de la rupture avec la tonalité et de la "dissociation" mahlerienne, qui nous permettent d'entendre autrement.

Cette discontinuité temporelle, qui constitue désormais le fond et l'enjeu du détail, doit être prise en charge par l'instance de ce qu'Adorno nomme, dans une belle formule, exacte Phantasie (699) : entendons par là " l'exactitude de la fantaisie imaginative ", dans la mesure où le mot d'“ imagination" renverrait encore au concept d'Einbildungskraft, qui, malgré sa richesse (kantienne par exemple), nous condamnerait à en rester aux attendus d'une philosophie de la conscience. Sans entrer ici dans les difficultés majeures et sous-évaluées encore de ce " champ de ruines " (Ricœur) que la philosophie nous a laissé en héritage sur le terrain de l'imagination, concevons que "la fantaisie imaginative " n'est évidemment pas le libre jeu d'une subjectivité débridée, mais le jeu de la finitude qui s'éprouve comme temporalité à la "mesure " de l'œuvre, comprise comme l'“écriture" exacte du temps. Une œuvre étant l'invention imaginative et rigoureuse d'une écriture du temps, c'est la même "fantaisie " qui " est à l'œuvre " et qui, comme art auditif du détail, “ouvre" le sens de l'œuvre qu'elle "déclôt” en s'y " attardant" : l'ouïr est donc une manière de retarder en s'attardant (verweilen), là où l'impatience fébrile se hâte vers la totalité. C'est dès lors la puissance de singularité irréductible du détail, auquel adhère une couleur singulière, qui peut être considérée comme le véritable lieu du musical. Ces détails sont comparables à des "sceaux", à des "noms", qui scellent pour nous l'authenticité d'une œuvre, tout en nous laissant irrémédiablement à distance, comme le confirment ces lignes extraites de Du mauvais usage du baroque : "Le rapport légitime aux œuvres d'art authentiques du passé, c'est la distance, la conscience de leur inaccessibilité, non l'empathie, qui tâtonne jusqu'à elles et leur fait injure par ses excès d'enthousiasmes". Ainsi, l'imagination est "capacité d'interpolation dans l'infime", comme le dit Adorno citant Benjamin; qu'elle soit créatrice ou auditrice, l'imagination étant créatrice jusque dans l'audition, elle seule peut plonger dans ce qu'est le devenir toujours obscur d'un art, en devenant elle-même Formphantasie - "fantaisie imaginative de la forme".

Ouïr la musique, c'est donc être capable d'entendre et de faire entendre, de remarquer et de porter à l'attention le "beau passage", qui n'a plus rien de ce qu'il peut habituellement signifier de cliché, mais qui est comparable, selon Adorno, à la citation vue par Walter Benjamin, ou pratiquée par Karl Kraus détournant des articles de presse. Dans un mouvement qui produit simultanément destruction et aura, le " beau passage" porte, en éclair, la lumière d'une beauté fragmentaire qui, lorsqu'on l'a perçue (wahrgenommen), ruine l'apparence dont la culture "recouvre" la musique: celui qui "entend" ainsi la musique ne peut que rompre avec les écoutes “ programmées " - par la culture, par l'historicisme, par l'esthétique, par l'industrie... À cet égard, les exemples qu'Adorno choisit et donne à entendre dans cette émission de radio, qui mènent de Bach à Schönberg, ont la grâce cursive de quelques instants mais aussi bien une force de "cristallisation " capable de faire entendre la musique contre les bien entendus des stéréotypes. Par exemple, il choisit, contre l'idéologie de la musique cosmique et close, contre la théologisation, contre les "légendes de l'historicisme ", de faire entendre combien est déjà présente chez Bach la plénitude du chant dans l'invention instrumentale. Chez Mozart, le beau passage peut être une interpolation qui surplombe toute connexion contextuelle et possède quasiment sa liberté: il peut même revendiquer la surprise de l'improvisé; le "musiquer" (Musizieren) devrait donc pouvoir s'entendre dans la force de son " commencement ", à 
même la manière dont le thème acquiert sa force d'expansion et son autonomie close. Même dans les grands opéras de Mozart, dit Adorno, il y a cette part qui reste dans l'ombre, se dérobant à la structure, jusqu'à disparaître. Adorno sollicite ses " auditeurs" : Vielleicht sind Sie empfänglich geworden? Lassen Sie nun... auf sich wirken... "Peut-être y êtes-vous désormais réceptifs? Laissez à présent agir sur vous..." L'entendre est encore une question, mais l'impératif est celui du ilassen ("laisser ") qui avoisine le logos de la phénoménologie : laissez la chose apparaître !

Ce qui peut alors être entendu est de l'ordre de la césure. Car les beaux passages ainsi compris, poursuit Adorno, seraient les équivalents de ce fameux moment des Affinités électives où Edouard et Ottilie, enlacés, voient se perdre toute espérance : Wie ein Stern fuhr die Hoffnung vom Himmel hernieder - "Comme une étoile l'espérance tomba du ciel...". Sans citer ici Benjamin, c'est à lui et à sa " critique " des Affinités électives que se réfère ici Adorno, lequel n'aura cessé, tout au long de son œuvre, de citer admirativement ce texte, parce que Benjamin y fonde son idée de la " césure " et parce qu'Adorno le juge comme l'essence paradigmatique de toute critique. De tels passages, qui sont donc les sommets de l'œuvre parce qu'ils portent en eux son invisible césure, sont, tout bien considéré, ceux qui étaient der Sprache der Musik beschieden, " donnés en partage à la langue de la musique ". Cette mise en œuvre concrète de la césure serait celle de la langue de la musique, qui vivrait au cœur des œuvres. C'est là-dessus qu'Adorno ne cesse d'attirer l'attention (Aufmerksamkeit) des auditeurs, en leur donnant à réentendre et à réécouter, comme chez Brahms ou Wagner, tel détail, telle cellule où se déposent la signification et l'impulsion " proprement " musicales. L'École de Vienne elle-même, même s'il est vrai qu'elle déroute les oreilles " culinaires " en supprimant la différence entre dissonance et consonance pour affirmer le primat de la construction, est capable de paradoxe : ainsi, chez Berg, chez Webern, se détachent aussi des instants qui, réactivant curieusement des valeurs expressives de la tonalité auxquelles leur musique donne une seconde vie, parviennent à une "suavité" d'expression où la musique est parfaitement "cristallisée". Ce programme peut dès lors s'achever, à propos de Schönberg, sur l'apologie de "l'oreille spéculative" (das spekulative Ohr). Tel est l'ouïr en effet : l'exactitude de la fantaisie imaginative dans le travail spéculatif de l'oreille, où l'œuvre autant que le " sujet " ne cessent de se déplacer et de se construire, dans l'épreuve de la finitude.

Cette "radiophonie" du "beau passage " montre que cette oreille spéculative est une oreille appareillée, mais qui n'oublie pas qu'elle est appareillée; qui n'utilise pas l'appareil contre sa finitude et contre son défaut d'oreille; qui n'en fait pas l'outil d'une prétention totalitaire à la totalité. La finitude de l'oreille appareillée, qui s'éprouve au contact de l'œuvre musicale et de sa technique, pose la question du sens de la technique : elle est le nécessaire et l'originaire supplément, qui comme technique de reproduction, technique de l'œuvre et productivité temporelle, permet à l'ouïr technique de décentrer radicalement le sujet de l'écoute. À l'inverse, l'industrie culturelle, qui instrumente cette technique, favorise le stéréotype et de l'œuvre et de l'écoute qui s'y prescrit - jusqu'au paradoxe où plus cette écoute subjective est singulière et plus elle est stéréotypée. C'est donc bien par la puissance productive de la technique et de l'appareillage qu'Adorno tente de faire pièce à cette instrumentalisation de la technique d'écoute par l'idéologie de la culture.

Ainsi, contrairement à ce que la vulgate a pu répandre, Adorno s'en prend aux prétendues vertus du live, pour faire expressément l'éloge du disque et de la radio, bref 
de l'enregistrement, parce qu'ils favorisent la fragmentation, l'imagination et la spéculation évoquées, c'est-à-dire la possibilité inédite d'une audition productive. Si les textes sur le disque sont désormais un peu mieux connus, le texte Über die musikalische Verwendung des Radios, "De l'utilisation musicale de la radio", qui n'est pas traduit, n'est jamais pris en référence, alors qu'il est un grand manifeste en faveur de l'appareillage de l'œuvre et de l'oreille. Adorno y soutient notamment que la technique radiophonique offre la possibilité de solliciter un erkennendes Hören, d'un oür capable de reconnaître la musique, en procédant par fragmentation et par "mise en pièces". Ainsi, écrit Adorno, contre l'idéologie de la grande musique, "la destruction de la symphonie à la radio est aussi un déploiement de la vérité ". Aussi bien, la radio relève des tâches d'un "musée musical", où enregistrement de nature "photographique", archives et haut-parleurs pourraient travailler contre la restitution auratique du live. Le thème de la répétition est ici central, tant du côté de l'interprète que du côté de l'auditeur, Adorno évoquant par exemple les soixante-dix répétitions demandées par Boulez pour Wozzeck. Dans ce parti pris radiophonique, la figure du chef d'orchestre disparaît pour laisser la place à celle du corépétiteur, qui est surtout un “interrupteur". Et c'est au cinéma, à la pratique filmique du cutters qu'Adorno emprunte l'idée du montage comme essentiel à l'ouïr. Il s'agit d'accuser césures et contrastes, pour corriger les clichés, en faisant de la radio comme on fait du cinéma. C'était déjà, dit Adorno, ce qu'Alban Berg avait en tête pour Wozzeck, opéra qu'il faut donc ouïr filmiquement et polyphoniquement. Si la notion d'écoute structurelle peut donc réapparaître in fine, c'est au conditionnel, pour dire une audition qui ne saurait plus être célébration sacralisante, mais coupe intégrant le commentaire et faisant saillir ce qui demeure en retrait dans la composition : bref, l'ouïr structurel demeure, mais selon une oreille différentielle, faisant le choix de la fragmentation et de la discontinuité contre le flux musical devenu le flux consommateur. La bonne oreille est celle qui se fait lectrice d'une écriture, dont elle sait qu'elle appartient à jamais à l'inachevé: la finitude est intégrée à ce travail musical qui doit se concevoir, dit Adorno, dans la lignée de ce que Schönberg avait tenté avec son Wiener Verein.

L'inachèvement, au cœur de l'œuvre, c'est son “ouverture" même, en ce qu'elle participe de la temporalité. Le temps est l'ouvroir: faisant éclater la fiction des totalités, des continuités et des identités, il déboute aussi un "sujet" qui se croirait assuré, en sous-main, de son “identité " d'écoutant, qu'il ramène à sa temporalité et à sa finitude. C'est d'ailleurs cette dimension du temps qui apparait encore dans le texte de Beaux passages, lorsqu'Adorno évoque l'origine del'échantillonnage des Beaux passages, qui ne relève de rien d'autre que de ce qu'il nomme das Zufällige des biographischen Schicksals, "le casuel de la destinée biographique". Il nomme par là l'enfance de l'oreille et les souvenirs musicaux "les plus précoces", die mir seit der Kindheit unvergesslich blieb, " qui, depuis, l'enfance me sont restés parce que je ne les ai pas oubliés". Le temps est à la source de toute "écoute" possible, parce qu'on (n') écoute (que) pour avoir déjà entendu. L'oreille spéculative et son différentiel reposent sur la mémoire et sur le souvenir, qui ouvrent la suite des temps et la possibilité de sa construction dans une écriture. Ainsi, dira Adorno, la musique est de nature scripturaire. Adorno traite de cette écriture dès 1934 dans La forme du disque, puis surtout dans Sur quelques relations entre musique et peinture, où il emploie deux mots, die Schrift (soit "la scription ")et l'écriture (écrit en français et en italique), pour dire l'articulation de deux temporalités, celle de l'immémorial et celle de l'histoire. Cette double écriture du temps, dont la construction est à la fois durée et discontinuité, se 
met en œuvre et s'“ entend" à partir de la césure. A l'œuvre, la temporalité finie déroute l'identité du sujet et de l'écoute qu'il déploie, si plastique soit-elle. Dans l'après-coup, le sujet s'avère aussi toujours en avant de soi, au défaut de soi ; et au lieu du défaut la musique donne à l'oreille la ressource d'une expérience productive du temps.

\section{Malaise dans l'écoute}

18 Cette articulation de la finitude, de l'œuvre et de la technique ramène donc l'“ écouter " à son "sujet" : la question "Qui écoute?" rebondit dans la mesure où l'écoute est l'affaire d'un "écouteur", qui, corollaire de l'invention de l'écoute technique, pourrait être aussi l'autre nom du " sujet " promu à son "oto-nomie ", lequel a trouvé un destin historique jusque dans la psychanalyse. Car Freud invente une écoute, et une technique d'écoute, au moment où le sujet va, en tous sens, "s'écouter", en même temps qu'il devient précisément l" écouteur".

On retrouve ici le cœur du " malaise " que Freud tente de décrire à propos de la culture et qui touche à la dimension du narcissisme, sur laquelle porte peu ou prou toute la seconde partie de son œuvre, bien au-delà du texte intitulé Pour introduire au narcissisme.. On retrouve aussi ce qui peut embarrasser dans la pensée d'Adorno, c'està-dire la difficulté à entendre le jeu du temps, de l'œuvre et de l'oreille. Si le destin métaphysique et historique de l'homme comme "sujet" est de devenir sourd à son propre défaut, il est celui de ce sujet qui se constitue comme identité dans l'illusion d'une écoute "pure", au nom de laquelle l'auditeur ou l'entendeur devient l'écouteur. L'écoute est le nom de ce virage et de ce malaise. La malaise dans l'écoute, qui est " malaise dans la culture", est celui d'une culture du sujet qui engage au narcissisme de l'écoute ou à l'écoute comme narcissisme: pareille écoute, idéalisée, essentialisée, serait comme le regard du sourd et l'" écouteur" serait à entendre en parallèle au voyeur. Ainsi, au voyeurisme pourrait répondre et correspondre un " écouteurisme ", qui, bien que le mot n'ait curieusement pas été créé, est un phénomène aussi essentiel que celui qui a trait au regard et participe autant que lui à la " condition de l'homme moderne". C'est par cet "écouteurisme" que passe la différence entre ce que nous avons nommé le "défaut d'oreille" et ce qui serait la schize de l'écoute. On pourrait ici reprendre les termes de Lacan et définir l'écoute sous le signe de l'objet $a$, à condition de souligner que l'ontologie de cette schize n'est pas celle de la finitude d'un défaut, mais, bien au contraire, celle qui procède de la toute-puissance de l'identité narcissique.

Une telle écoute est autarcique, tautologique; elle fonctionne en réverbération, selon l'excès d'une identité saturée. Mais parce qu'elle touche au narcissisme, elle suppose aussi que le sujet de l'écoute est toujours à double fond. D'un côté, il est celui de la jouissance hédoniste de soi, de l'immédiateté du “j'ouïr", qui s'éprouve là où l'on fait les enfants par l'oreille. De l'autre, ce sujet est sous l'emprise secrète d'une puissance qui joue un rôle décisif dans l'écoute, c'est-à-dire le Surmoi, qui, favorisant la posture de "l'être à l'écoute " - comme on est " aux aguets " et comme on est " aux écoutes " nous destine à l'angoisse du bouche à oreille et du ouï-dire. Ce qui suture ces deux faces du sujet, c'est la menace de la dissociation de nature schizophrénique, qui guette le sujet moderne. Cette dissociation peut être celle de la "distraction", dont l'étymologie et l'histoire lexicale nous permettent de réunir trois sens et trois phénomènes : celle de la torture, c'est-à-dire du démembrement des corps, celle de l'éclatement interne du sujet 
schizoïde et celle de l'éclatement du "je" dans le divertissement. Dans la dissociation du sujet rôde et triomphe ainsi une culture de la pulsion de mort. L'écoute narcissique est celle qui est en prise sur la force léthale de la pulsion.

La mort est dans l'écoute, en effet : comme pulsion et comme nirvana dirait Freud. Au lieu de la pulsation, du rythme et de la discontinuité temporelle. Cette force de la pulsion de mort rencontre, sur le régime du temps, l'impossibilité de la discontinuité, l'impossibilité de la continuité du discontinu, et se renforce donc du flux et de son alliance avec la dissociation atomistique. L'écoute correspondant à ce malaise dans l'écoute est celle d'un sujet dont la temporalité ne peut se construire. Aussi, le sujet "s'écoute", dans l'illusion de sa durée, et en plusieurs sens. Il s'entend parler, en quoi l'autarcie narcissique se relie à la paranoïa du surmoi : c'est là que peut surgir le lapsus, le saut de la discontinuité forcée sur le théâtre rhétorique, là où l'écouteurisme triomphait dans l'illusion de la continuité éloquente. Lorsqu'il "s'écoute ", c'est-à-dire qu' il s'écoute trop", le sujet est aussi " en souffrance ", la souffrance narcissique le condamnant à "s'écouter" et cette écoute participant de sa souffrance. Enfin, il y a dans ce narcissisme de l'écoute, comme un auto-érotisme, dirait Freud, une réponse narcissique à l'écho de l'unité et du démembrement qui s'opèrent dans l'acte sexuel. Voilà qui nous reconduit au phénomène de la schizophrénie et à la "machine à influencer" de Tausk : l'Écho (de Narcisse) est comme un objet interne, lié au Surmoi, qui peut être écouté comme une menace provenant des bruits internes du corps, et que le sujet tente d'étouffer.

Le texte de Freud L'homme aux rats, l'une des célèbres Cinq psychanalyses, montre que le sujet qui "s'écoute “s'entend parler: c'est l'émergence de l'inquiétant familier, la présence terrifiante de la phoné sous le signifiant "RAT" - dont Freud fait la métaphore du signifié. À partir de L'homme aux rats, Freud renverse les propositions qu'il avait avancées sur le vu et l'entendu dans L'interprétation du rêve : dans ce texte ci, la psychose hallucinatoire visuelle est assimilée au rêve; elle fait valoir la présence de l'image, la régression de la pensée à l'image, du mot à l'investissement de la chose. Inversement, avec le texte de l'Homme aux rats, il apparaît qu'à force de voir on écoute, et qu'à force d'écouter, on hallucine visuellement. L'obsession dit autant que le délire, et le délire autant qu'une voix. On entend la voix délirante du langage, l'ordre sans ordre, la voix sans parole, à laquelle l'oreille de l'homme obéit. Telle est la source du ouï-dire, du jeu de l'obédience et de l'obéissance, où le langage, écho d'écho, tourne à “l'échologie". La "suffisance" de l'écoute, de qui trop s'écoute, donne au langage sa force d'autorité, là où le vide de la discontinuité n'a plus sa place ni sa fonction. Ce modèle d'écoute est branché sur l'écoute conjuratoire du danger interne (pulsionnel), où l'écouteur est le sujet au miroir, qui n'investit l'écoute que dans le simulacre et la mimique.

Aussi, qui écoute s'adresse à l'écoute, c'est-à-dire à celle du langage comme tel, auquel il prête l'oreille comme à une phonè inlassable, qui ne cesse de faire entendre la mort en sa pulsion et qui déclenche la terreur émanant de la puissance du Surmoi. C'est la voix de "son maître", dont le pouvoir se régale, puisqu'il est emprise sur l'inconscient du sujet, en effet assujetti. Le redoublement de la phonè dans la voix n'est toujours qu'un ouï-dire, et la terreur qu'elle peut susciter dans le langage ne peut être qu'assimilée à la conscience et au Surmoi, qui fondent une écoute de surveillance - " aux aguets ". Dès lors, si subjective et si plastique soit-elle, une telle écoute ne peut que maintenir, voire renforcer l'autorité là où l'on prétend la faire disparaître: ainsi, vouloir faire 
disparaître l'œuvre et sa temporalité condamne en réalité à raffermir le pouvoir totalitaire des codes et de l'institution.

Inversement, ouir, au défaut d'oreille, contre le clivage de l'écouter et de l'entendre, contre le clivage de l'auscultation et de l'intellect, dirait une "écoute " qui ne serait pas branchée sur la pulsion de mort, mais sur la pulsation de la mort: soit sur une temporalité. Ouïr, au défaut d'oreille, dirait une "écoute " où la musique, la parole, la voix ne seraient pas non plus clivées par la fiction du son pur, et pourrait réinstancier une écoute où musique, parole et voix s'articuleraient sur le silence. Ouïr, au défaut d'oreille, dirait une "écoute" allant de pair avec le silence, parce qu'il participe de la construction du sens, et parce que sans lui l'écoute devient une idéalité ou un absolu, comme dans le phénomène que nous avons nommé l'écouteurisme. Le silence, c'est l'apparition d'un foyer de nudité dans le langage, c'est une anankè, sans laquelle nous entrons, pour le langage comme pour le "son ", dans la folie de l'“ en présence ", qui gage toutes les audiences, les obédiences et les obéissances.

Oür, c'est admettre le jeu de l'inconscient comme force de la finitude : contre toutes les formes d'“ échologie ", le fameux chaînon manquant entre soma et psychè permet aussi les syntaxes et les articulations. Au défaut de l'unité et de l'identité de l'écouteur. On sait, avec Freud, que l'unité du corps est à perdre (et même celle du corps social), et elle est recouvrée en public: personne ne peut se dire le membre ou le garant de cette unité. Une obéissance à la voix du passé (comme par exemple avec Eॄdipe, entendant, écoutant la parole de l'oracle) n'est pas obéissance à la voix de la structure autoritaire. Parce que l'inconscient nous relie à la mort et à son silence, avant même l'ouverture de la bouche et de l'oreille. Et là seule la musique, peut-être, est en mesure, dans la langue, de faire entendre la mort. Contre la hiérarchie que nous impose l'écoute, contre le logos narcissique d'une voix sans parole, qui est aliénation, il faut jouer l'incarnation $\mathrm{du}$ verbe et trouver la fonction de la parole. Alors, le son fait voir (Artaud) : vibration, onde, rythme peuvent l'emporter sur l'idée d'image acoustique. Il y a là un pouvoir d'expérience, un "écouter voir", où le sujet est "en imagination", en avant de soi. C'est précisément à l'œuvre de travailler à cette image du temps: en décentrant le sujet, dont l'écoute est alors le temps de lire en soi-même. Qui écoute lit le temps. À même les œuvres. Si l'ouïr peut être "structurel ", c'est, dit Adorno, à la mesure d'une teneur de vérité des œuvres qui est à la fois indispensable et jamais définitivement donnée. Cette vérité est temporalité et finitude, elle est en mouvement, et c'est l'œuvre elle-même qui prépare le processus temporel de son " écoute ".

\section{Ouïr à l'envers}

Proust expose, "comme personne", ce qu'est "l'écoute" à l'œuvre, parce que La Recherche du temps perdu, à la fois théorie du temps, théorie de son écriture et théorie de l'œuvre d'art, est aussi une théorie de l'ouïr et du voir qui engage moins une définition du sujet que celle de sa temporalisation. Qui écoute, en effet, la Sonate de Vinteuil ? À la lettre, personne. Le mot "écoute " est absent de la Recherche et le verbe "écouter " y est peu fréquent. Mais le sujet qui serait en position d'écoute est absent lui aussi : parce qu'il est sourd ; parce qu'il est toujours autre et se métamorphose, passant notamment du personnage de Swann à celui de Marcel ; parce qu'il ne peut entendre qu' "en avance " sur lui-même et en changeant au fil des auditions successives; parce qu'il est finitude radicale, par sa temporalité même. Mais en (n') étant personne, le " qui " de 
“l'écoute ", à l'épreuve du "quoi", devient enfin quelqu'un : parce que l'œuvre est un appareillage, c'est-à-dire un instrument de reproduction, qui peut seul produire la dimension et la vérité de la première fois. La sonate de Vinteuil est le nom de l'œuvre, c'est-à-dire l'histoire de sa reproduction et de sa transmission, qui permet le déploiement des différentiels temporels de l'ouïr. Sept épisodes - chiffre névralgique lui sont consacrés, qui correspondent en réalité à huit auditions : tout se joue dans ce décalage entre sept et huit, qui correspond à cet instant qui a déjà eu lieu sans avoir eu vraiment lieu, qui a besoin de la seconde fois pour être une "première fois", qui ne serait rien sans sa répétition, sa reproduction ni sa modification - décalage qui creuse, à même l'instant, ce boitement de l'avance et du retard définissant la finitude et la temporalité dont l'ouïr est un décisif enjeu.

Dans le premier épisode, en effet, lors de la soirée chez Madame Verdurin, une œuvre est jouée au piano, et personne n'entend rien. Swann n'écoute pas. Rien n'est donné à entendre au lecteur. Mais l'audition a néanmoins une réalité décisive, car elle fait surgir ce qui est une première fois alors oubliée : "L'année précédente, il avait entendu une œuvre musicale exécutée au piano et au violon." Ainsi, la première audition n'apparaît que dans l'après-coup de la seconde fois, qui n'est elle-même possible que parce qu'il y a eu cette première fois. La deuxième fois est donc l'occasion de "l'avoir entendu ". Et ce qui se transcrit d'abord, c'est ce qui revient à Swann, l'impression inscrite dans le " fac-similé " de la mémoire, la "transcription ", dit Proust, c'est-à-dire l'enregistrement et l'arrangement d'une écriture. De l'écriture d'un fragment: “Une phrase". Cette reproduction prépare le premier ouïr : non seulement parce qu'elle est transcrite, mais aussi parce qu'elle est transposée dans l'univers esthétique et amoureux de Swann, qui développe à partir de la musique un idéalisme musical, avant d'oublier l'œuvre et sa trace. La seconde fois, dans ce même épisode de la soirée Verdurin, où joue le "petit pianiste", permet l'apparition de la phrase et sa nomination: "On lui dit que c'était l'andante de la Sonate pour piano et violon de Vinteuil", mais l'audition est aussitôt recouverte par l'incompréhension de l'écoute culinaire et par le ouï-dire sur l'aliénation mentale de Vinteuil.

Le deuxième épisode, donc la troisième audition, a lieu chez Verdurin encore. Swann et Odette sont ensemble. Écoutant le pianiste, ils s'attachent à la " petite phrase ", dont ils font "l'air national de leur amour". Pourtant, Swann, par une comparaison picturale (Pieter de Hooch), ménage l'ouverture de la musique sur un autre monde. Ouverture ambiguë : il verse dans l'idolâtrie, mais la musique entendue garde intacte " une beauté intrinsèque ".

$\mathrm{Au}$ cours du troisième épisode (quatrième audition), Swann, chez lui, fait jouer Odette, “ bien qu'Odette jouât fort mal, mais la vision la plus belle qui nous reste d'une œuvre est souvent celle qui s'éleva au-dessus des sons faux tirés par des doigts malhabiles d'un piano désaccordé ". La musique est ici l'occasion du passage de l'entendre à l'écouter, et surtout de la naissance, en Swann, de l'écouteur! "Mais la petite phrase, dès qu'il l' entendait, savait rendre libre en lui l'espace qui pour elle était nécessaire, les proportions de l'âme de Swann s'en trouvaient changées ". L'écoute est ici jouissance, éveil, vacance, anesthésie : “À voir le visage de Swann pendant qu'il écoutaitla phrase, on aurait dit qu'il était en train d'absorber un anesthésique." L'écoute est ici décrite littéralement dans la jouissance de l'illusion d'un son pur. Swann se transforme en une créature étrangère à l'humanité, une créature chimérique ne percevant le monde que par l'ouïe. "Quelle étrange ivresse il avait à dépouiller son âme la plus intérieure de 
tous les secours du raisonnement et à la faire passer seule dans le couloir, dans le filtre obscur du son!" Ainsi, la continuité répétitive et obsessionnelle se marque dans cette demande musicale et amoureuse de Swann envers Odette, qui doit "rejouer dix fois, vingt fois ", et qui doit l'embrasser en même temps.

Le quatrième épisode, pour la cinquième audition, se déroule lors de la soirée chez Madame de Saint-Euverte, où Swann est seul. L'œuvre est exécutée au piano et violon ; à l'apparition de la petite phrase répond l'aveuglement de Swann, superposant, tel Schopenhauer, la musique à l'expérience de la douleur et à sa consolation. Cette " cécité" idéalisante, qui conduit Swann à s'identifier à Vinteuil, ouvre néanmoins sur la temporalité. Swann revient sur l'année écoulée, et "après la soirée Verdurin, se faisant rejouer la petite phrase ...", il mesure enfin la dimension d'un écart qui est à la fois temporel - l'écart entre aujourd'hui et la première fois - et musical - "le faible écart entre les cinq notes qui la composaient", avec l'accent mis sur le rappel de deux d'entre elles. Swann change de plan : "Il savait que le souvenir même du piano faussait encore le plan dans lequel il voyait les choses de la musique, que le champ ouvert au musicien n'était pas un clavier mesquin de sept notes, mais un clavier incommensurable..." L'œuvre ouvre alors sur la dimension de la contradiction temporelle entre l'œuvre et ce vide "que nous prenons pour du néant". Comme tel thème de Tristan, la petite phrase devient l'enjeu d'une mémoire latente, qui engage la question de la survivance et de la mort, bref celle de la finitude. Swann serait à la limite d'entendre, enfin: "Elle avait disparu. Swann savait qu'elle reparaitrait à la fin du dernier mouvement (...) Il y avait là d'admirables idées que Swann n'avait pas distinguées à la première audition et qu'il percevait maintenant comme si elles se fussent, dans le vestiaire de sa mémoire, débarrassées du déguisement uniforme de la nouveauté. Swann écoutait..." Mais il entend à nouveau, dans le dernier mouvement, le "dialogue entre le piano et le violon " : alors surgit la métaphore de l'arc-en-ciel et du prisme. Swann écoute mais n'entend pas. Il redevient Swann, ce "célibataire de l'art", qui replonge dans ses études esthétiques et finalement dans son éternelle "procrastination".

31 Dans A l'ombre des jeunes filles en fleurs, lors du cinquième épisode, soit lors de la sixième audition, Mme Swann se met au piano pour jouer la partie de la Sonate de Vinteuil où se trouve la petite phrase que Swann avait tant aimée. Relayant le personnage de Swann, celui de Marcel entre désormais en scène, pour reprendre et commenter la dimension du temps qui s'inscrit comme phénomène-clef dans le jeu de l'écoute et de l'audition. L'enjeu, c'est le sens de l'expression "la première fois ": “ Mais souvent on n'entendrien, si c'est une musique un peu compliquée qu'on écoute pour la première fois. Et pourtant quand plus tard on m'eut joué deux ou trois fois cette sonate, je me trouvai la connaître parfaitement. Aussi n'a-t-on pas tort de dire "entendrepour la première fois ". Si l'on n'avait vraiment, comme on l'a cru, rien distingué à la première audition, la deuxième, la troisième seraient autant de premières, et il n'y aurait pas de raison pour qu'on comprît quelque chose de plus à la dixième. Probablement, ce qui fait défaut, la première fois, ce n'est pas la compréhension mais la mémoire." Défaut de mémoire, donc, mais formation du souvenir : tel est le fond de toute audition possible, compliquée du fait qu' "on ne perçoit d'abord que les parties les moins précieuses", d'où naît le sentiment "de la mélancolie qui s'attache à la connaissance de tels ouvrages, comme de tout ce qui se réalise dans le temps". La finitude du percevoir n'est plus mélancolique désormais, parce qu'elle engage une vérité qui est tout à la fois celle de l'œuvre et celle de la vie: Certes, dit Marcel, “je ne la posséderai jamais tout 
entière : elle ressemblait à la vie...", mais il y découvre la ressource majeure d'un différé qui devient paradigmatique de ce qu'est l'enjeu de l'œuvre d'art : “Ce temps du reste qu'il faut à un individu - comme il me le fallut à moi à l'égard de cette Sonate pour pénétrer une œuvre un peu profonde, n'est que le raccourci et comme le symbole des années, de siècles parfois, qui s'écoulent avant que le public puisse aimer un chef d'œuvre vraiment nouveau." Ainsi, ce qu'on appelle la postérité est la postérité de l'œuvre, au sens où il faut que l'œuvre crée elle-même sa postérité. Dès lors, dit Marcel, “je fus ravi d'entendre jouer Madame Swann".

Dans La Prisonnière, le sixième épisode - la septième fois - est une soirée chez les Verdurin. Il commence par une surdité et une méprise : “Je crus qu'on commençait par des œuvres d'autres musiciens que Vinteuil. Car je croyais qu'on ne possédait de lui que sa Sonate pour piano et violon." Un pays inconnu s'ouvre, une musique nouvelle apparaît, mais Marcel finit par retrouver la Sonate de Vinteuil, "l'accent si amicalement connu", qui paraît indiquer un chemin. "C'était une œuvre inédite de Vinteuil", avec un jeu d'allusions à la petite phrase. La joie ressentie est celle du commencement et de la promesse, qui se traduit dans la couleur rouge des sept notes et dans la figure de l'Aurore, avec une composition qui se déploie et s'accomplit désormais comme Septuor. La nouveauté de cette œuvre, qui est celle de la musique de Vinteuil, se mesure dans la comparaison et dans l'écart entre la phrase et ce Septuor, et laisse découvrir, "fragmenté par les lacunes que laissaient entre elles les auditions successives", un univers qui vit précisément d'une écriture de la répétition : la reconnaissance du chant est possible grâce à ce travail de "transposition, dans l'ordre sonore, de la profondeur". Une phrase de la Sonate revient dans le Septuor, mais chaque fois changée, elle est "la même et pourtant autre, comme reviennent les choses de la vie ". Le rouge peut être l'écarlate de la robe d' " un archange de Mantegna sonnant du buccin", qui annonce la joie de la révélation. Mais au prix d'une autre révélation et d'une autre profondeur : si cette musique de Vinteuil a pu être entendue, en effet, et alors qu'on croyait que tout ce qui n'était pas la Sonate "demeurait inexistant en d'indéchiffrables notations ", c'est parce qu'à l'inverse de ce qu'on aurait attendu, l'amie de la fille du compositeur a réussi à "débrouiller le grimoire" et les " hiéroglyphes" que celui-ci avait laissés. Ces deux jeunes gomorrhéennes n'avaient apparemment, à l'égard du musicien, que sentiments de sadisme et désirs de profanation, mais c'était en allant "à l'opposé de [leurs] inclinations véritables...". Ainsi, l'inversion est la seule manière de préparer le déchiffrement d'une écriture qui annonce, pour l'auditeur, l'Ange écarlate du matin, “l'étrange appel " d'une promesse.

Ce sera tout le sens du septième et dernier épisode, huitième audition, lorsqu'Albertine joue la musique de Vinteuil au pianola. Par un apparent paradoxe, c'est un instrument de musique mécanique qui permet à Marcel, soucieux de se faire rejouer successivement les morceaux, de modeler "une nébuleuse encore informe" et de donner enfin "un volume à la musique ". Mais ces rouleaux, qu'Albertine demande à Françoise de changer, sont bien de la musique incisée, c'est-à-dire la conjonction d'une écriture et de la reproductibilité instrumentale. Grâce à Albertine l'invertie, et les possibilités que lui offre une écoute inversée, Marcel découvre la musique, dans le phénomène de la "vraie transmutation" d'un état à l'autre, ou dans celui de la tardive apparition de "ces phrases identifiées avec des phrases des autres œuvres, comme cette phrase de la Variation religieuse pour orgue, qui, chez Mme Verdurin, avait passé inaperçue pour moi dans le septuor... " Ouïr, c'est donc aller de "la déception que cause d'abord un chef d'œuvre" à ce qui devient l'apanage de "l'imagination", qui peut 
s'ouvrir à "la fête inconnue et colorée (dont ses œuvres semblaient les fragments disjoints, les éclats aux cassures écarlates)". Le pianola est ainsi comme une "lanterne magique", qui projette en fait l'image même d'Albertine, transfigurée en ange musicien, en sainte Cécile, mais surtout en "grande déesse du Temps" : car la musique n'est pas le dernier mot à ce stade de La Recherche, il faut encore apprendre à "ouïr " le temps lui-même à l'envers pour que l'écriture le fasse advenir à son commencement.

De la Sonate au Septuor, c'est la temporalité toujours redoublante de celui "qui écoute" qui prend racine et forme dans la répétition et par la mécanique de la reproduction. Le temps proustien est un temps pensé, qui n'a plus rien du temps des philosophes et n'est plus la fiction de ce temps linéaire qui condamne à la mélancolie du passage, à l'illusion de l'instant ponctuel et aux erreurs sur la mémoire. La temporalité porte en elle la reproductibilité comme source de l'avenir, parce que l'instant est toujours déjà double, et comporte essentiellement une part non vécue, un "temps perdu" qui demeure en attente de son devenir et de son écriture. Cet instant fracturé, qui jamais ne se superpose à lui-même, est aussi la fracture du sujet et de son “ouïr", en ruinant d'avance toute possibilité d'identité. La finitude et la mort passent par cette fracture, et conditionnent ainsi une construction du temps qui est sa "reprise", infinie et toujours ouverte. Cette temporalité ne va dès lors cesser de creuser la "contradiction incompréhensible de la survivance et du néant", qui s'entrecroisent dans l'expérience de Marcel et dans celle du narrateur. "La brusque révélation de la mort avait, comme la foudre, creusé en moi... comme un double et mystérieux sillon." Ce sillon, ce micro-sillon, ouvre à l'écoute du temps, puisque " notre moindre désir, bien qu'unique comme un accord, admet en lui les notes fondamentales sur lesquelles toute notre vie est construite..." Mais encore faut-il entendre l'appel, comme on entend ou n'entend pas l'appel du Septuor. Plus encore : entendre l'appel ne suffit pas, car il ne crée pas les forces, pas plus qu'il ne fait de Swann l'écrivain qu'il n'était pas. De fait, celui qui écoute ne sait pas souvent ce qu'il écoute, et ne sait pas davantage qui il est, c'est-à-dire un créateur en puissance, puisque toute vie est aussi une œuvre d'art à construire. "Comme si nos plus belles idées étaient comme des airs de musique qui nous reviendraient sans que nous les eussions jamais entendus, et que nous nous efforcerions d'écouter, de transcrire." Il faut encore être capable de "bien lire en soi-même ", c'est-à-dire de lire à l'envers les sillons du temps toujours déjà perdu. Lire à l'envers et ouïr à l'envers, puisque tout ce qui ouvre et crée l'avenir dans $L a$ Recherche fonctionne sur le régime de l'inversion et " marche en sens contraire" - depuis Bergotte qui "parle à contresens " et Elstir qui " peint la mer par l'autre sens", jusqu'aux figures de l' “inversion sexuelle”, "phénomène si mal compris, si inutilement blâmé".

Ainsi, à l'image dela photographie, si décisive dans La Recherche du temps perdu, la musique délivre du temps, délivre le temps, parce qu'elle reconduit le narrateur et le lecteur à la finitude de l'instant, qui demeure en attente non de sa réécriture, mais de ce qui sera dans l'écriture le véritable développement de son négatif. Ainsi, à l'image de la musique, l'œuvre devient l'original du temps, sa production originale. Où la mort évidemment programmée s'entend à distance. Et s'accomplit. Proust l'entend venir, et peut mourir quand l'œuvre est achevée - dans son inachèvement même. 